\title{
How Does the Multi-Disciplinary Team Impact Chronic Kidney Disease Management?
}

\author{
Baoying Huang ${ }^{1}$, and Michael Fawzy ${ }^{2, *}$ \\ ${ }^{1}$ Medical Student, Anglia Ruskin Medical School, UK \\ ${ }^{2}$ Renal Consultant, Basildon University Hospital, Basildon, UK
}

*Corresponding author: Michael Fawzy, Renal Consultant, Basildon University Hospital, Basildon, UK, E-mail: michael.fawzy@nhs.net

Received: 04 Jul, 2019 | Accepted: 26 Jul, 2019 | Published: 31 Jul, 2019

Citation: Huang B, Fawzy M (2019) How Does the Multi-Disciplinary Team Impact Chronic Kidney Disease Management? Int J Nephrol Kidney Fail 5(2): dx.doi.org/10.16966/2380-5498.177

Copyright: (C) 2019 Huang B, et al. This is an open-access article distributed under the terms of the Creative Commons Attribution License, which permits unrestricted use, distribution, and reproduction in any medium, provided the original author and source are credited.

\begin{abstract}
Chronic Kidney Disease (CKD) is a condition where a gradual kidney function loss leads to a build-up of toxic waste products in the blood, increasing morbidity and premature deaths from enhanced risks of cardiovascular disease, stroke and mineral bone diseases. The most common causes of CKD include type 2 diabetes and arterial hypertension, both accounting for two-thirds of all cases. CKD is asymptomatic in early stages and is only diagnosed via routine screening tests unless severe symptoms arise from the advanced CKD. Management of CKD via Multidisciplinary Team (MDT) consisting of professionals from various disciplines has been hailed as the best modality in treating such a multi-faceted chronic disease, and it has been shown to have positive impacts on patients' experience and clinical outcomes.
\end{abstract}

\section{Introduction}

More than 1.8 million people are diagnosed with CKD in England [1] where the advancing age is commonly associated with the higher incidence of CKD stage 3-5 (Figure 1), is an illustration that on average, although only $1.9 \%$ of the population is estimated to be having moderate to severe CKD, that figure is increased to $32.7 \%$ for those aged 75 and over. The latest guideline established that NHS England spent $£ 1.45$ billion in $2009-10$ attributing to $£ 1$ in every $£ 77$ spent by NHS in managing CKD and associated illnesses stemmed from the condition [1]. Progression patterns and risk factors of CKD into End-Stage Kidney Disease (ESKD) can be established well before the need to initiate Renal Replacement Therapy (RRT). Therefore, proactive intervention strategies to prevent its progression form a basis of management of CKD which is mainly delivered via a MDT to address all the various aspects and risk factors of CKD. This paper aims to explore and describe how effective this MDT approach is in treating CKD.

\section{Chronic Kidney Disease (CKD)}

Chronic kidney disease (CKD) is a multi-factorial disease leading to progressive worsening of kidney function. Kidney biopsies from most CKD patients will often show glomerulosclerosis, tubular atrophy and interstitial fibrosis. Moreover, at the end stage of kidney failure, they will feature a characteristic renal fibrosis where the kidney undergoes unsuccessful wound healing after chronic and sustained inflammation and injury to its tissues. CKD can be classified into 5 stages based on an Estimated Glomerular Filtration
Rate (eGFR) which represents the amount of fluid each functioning unit (nephron) of the kidneys filters through, per unit time and thus acts as an indicator of kidney function. Figure 2 demonstrates the progression of CKD in stages using eGFR and albumin to creatinine (ACR) ratios [3]. A consistently high level of kidney disease markers (illustrated in table 1) over a period of 3 months and a GFR at G3 stage are two criteria that need to be met in diagnosing CKD. G5 of GFR is termed as End-Stage Kidney Disease (ESKD) where kidney failure can only be managed with Renal Replacement Therapy (RRT) including dialysis or kidney transplant unless the patient wishes to undergo conservative management.

\section{Importance of estimating Glomerular Filtration Rate (GFR) in diagnosing $\mathrm{CKD}$}

Glomerular Filtration Rate (GFR) is regarded as a marker of renal function and can be determined by plasma clearance of filtration markers which are typically not metabolised and reabsorbed in the kidneys and are therefore physiologically inert, and are of a low molecular weight to be filtered by the glomeruli [4]. Candidate filtration markers include exogenous inulin, iothalamate, Ethylenediaminetetraacetic Acid (EDTA) and iohexol and endogenous creatinine and cystatin C [5]. The "gold standard" GFR measurement involves using exogenous inulin as a filtration marker which is a $5200 \mathrm{Da}$ polysaccharide produced by root plant tubers [6]. Inulin clearance has the greatest diagnostic sensitivity of detecting CKD in early stages since it is exogenous and filtered unchanged at the glomerulus without getting reabsorbed in the renal 


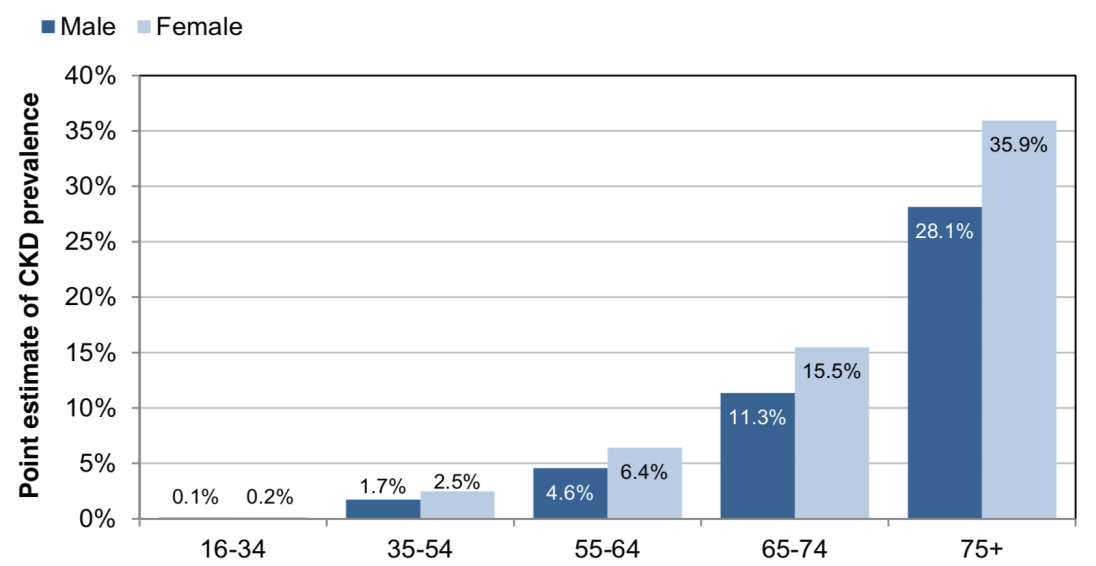

Figure 1: A chart to illustrate the expected prevalence of CKD at Stage 3-5 in England in both males and females [2].

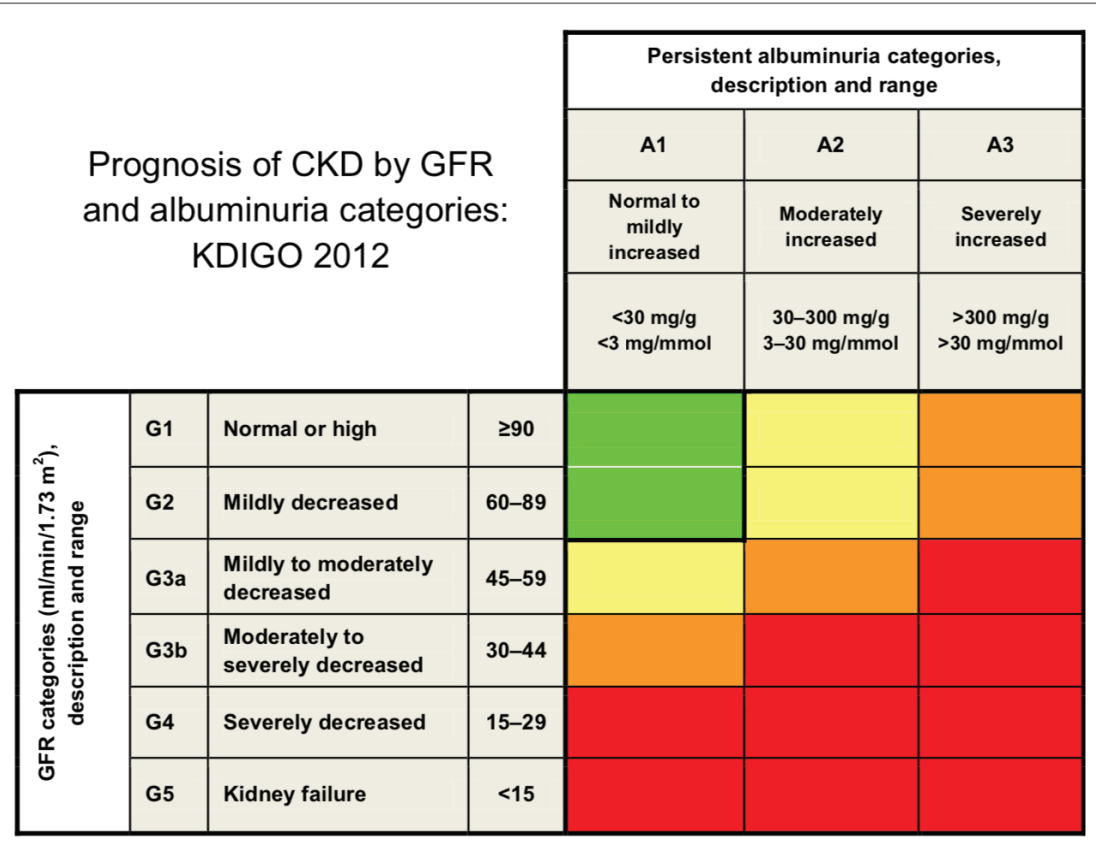

Figure 2: Chronic Kidney Disease (CKD) can be classified according to patients' Glomerular Filtration Rate (GFR) levels and their levels of risk can be based on albumin to Creatinine Ratio (ACR) [3].

tubules and thus used as a benchmark in comparing newer GFR measurement methods. However, measuring urinary inulin clearance is tedious, involving fasting of subjects, continuous intravenous (IV) infusion starting with priming dose, repeated blood and urine sample collection in a timely manner [7] and therefore is not as widely used in most laboratories.

Creatinine, instead, is the most widely and commonly used filtration marker. It is a biological waste product from creatine phosphate formed during wear and tear of muscles and excreted unaltered via glomerular filtration in the nephrons of kidneys. Once kidney function decreases, its rate of creatinine filtration also decreases, allowing serum creatinine levels to rise. Serum creatinine concentration alone cannot be used as an indicator of glomerular function as they are dependent on one's muscle mass, age, ethnicity, diet and gender [8]. Therefore, it can be inferred that a 25-year-old African-American male of a strong build and a lean 75-year-old female of White ethnic origin, will not have the same GFR even if they both shared the same serum creatinine levels. In these circumstances, it is paramount that such variables can be factored in appropriately when estimating GFRs from serum creatinine levels, leading us to the development of various Isotope Dilution Mass Spectrometry (IDMS) equations, most notably, the Modification of Diet in Renal Disease (MDRD) Study equation and the Chronic Kidney Disease Epidemiology Collaboration (CKD-EPI) equation which needs stable GFR for proper calculations [9].

\section{Causes and risk factors of CKD}

Exact aetiology of CKD is not fully understood; however, older age, Diabetes Mellitus (DM), hypertension and obesity are associated 
Table 1: Markers commonly used to diagnose kidney damage. Presence of one or more of these kidney markers along with the consistent decrease in GFR is required in diagnosing CKD [24].

\begin{tabular}{|l|}
\hline \multicolumn{1}{|c|}{ Kidney Disease Markers } \\
\hline $\begin{array}{l}\text { Albuminuria (based on ACR levels of more than } 30 \mathrm{mg} / \mathrm{g} \text { or } 3.39 \mathrm{mg} / \\
\mathrm{mmol})\end{array}$ \\
\hline $\begin{array}{l}\text { Abnormalities in urinary sediments including red and white blood cell } \\
\text { casts, oval fat bodies and granular casts }\end{array}$ \\
\hline Electrolyte abnormalities \\
\hline Pathological findings in histology \\
\hline Abnormal structural findings in imaging of kidneys \\
\hline Previous history of kidney transplantation \\
\hline
\end{tabular}

with diabetic glomerulosclerosis and hypertensive nephrosclerosis both leading to CKD in Western world [10]. Main causes of CKD in developing countries include glomerular and tubulointerstitial diseases due to infections, drug and toxin exposure. A small percentage of CKDs also arises from congenital conditions such as polycystic kidney disease. In addition, autoimmune causes also play a great role in CKD since IgA nephropathy is one of the most common causes of CKD after DM and hypertension [11]. Gender difference plays a role in CKD with males showing a more rapid and aggressive CKD progression, suggesting the potential roles of sex hormones in modulating synthesis of growth factors and chemical mediators leading to CKD progression [12].

Ethnicity and socioeconomic status are also key modifiers in CKD prevalence and progression. There is a $60 \%$ increased risk of worsening CKD in people of the lowest social quartile when compared with their richer counterparts. This progression is highest in people with ethnic minority backgrounds and can be attributed to having reduced medical awareness and access to specialist nephrologist care in both pre-dialysis and dialysis groups [13]. Major risk factors of CKD include DM, hypertension, obesity, family history of CKD, age and gender. Obesity is one of the strongest modifiable risk factors leading to CKD since it carries a highest risk of leading to glomerular hypertrophy and focal and segmental glomerular sclerosis leading to ESKD [14]. Kidney injury and renal function decline from increasing glomerular capillary pressure and decreasing density of podocytes from glomerulomegaly and hyperfiltration in obese patients often lead to the development of CKD in the obese [14].

\section{Clinical presentation and complications of CKD}

$\mathrm{CKD}$ is non-symptomatic in the earlier stages and most diagnoses are made via routine tests unless patients present with severe symptoms from advanced CKD. As the kidney gradually loses its function from progressive disease, there is a rapid accumulation of uremic retention solutes in the body which can then affect various parts of the body. Uremic toxicity can lead to vascular damage increasing the risk of cardiovascular diseases and bleeding episodes, impaired inflammatory and immune responses, and altered microflora in the digestive system [15]. Impairment to normal kidney functions can lead to proteinuria, oedema, hypertension, hyperphosphatemia, hyperkalaemia, anaemia and bleeding diathesis, renal osteodystrophy, congestive heart failure, gastrointestinal disturbances and generalised myopathy [16].

\section{Multi-disciplinary team in management of CKD}

With medication compliance and adequate lifestyle changes including; keeping a normal weight, taking up exercise and following a renal diet; CKD patients can remain as healthy as possible. Management plan is aimed to treat primary pathological diagnosis along with an intervention based on eGFR stages and albuminuria to control hypertension, diabetes, dyslipidemia and anaemia to prevent episodes of acute kidney injury (AKI) and reduce its complications. In Latin America, where the ESKD prevalence has increased from 119 patients pmp (patients per million of population) in 1991 to 660 pmp in 2010 pmp, with Puerto Rico, Argentina, Mexico, Uruguay and Chile reporting the highest rate of patients undergoing RRT between 777 and 1135 pmp [17]. Most CKD patients are managed by doctors specializing in internal medicine, with a nephrologist referral reserved to those with advanced stage CKD. However, in the UK, NICE has recommended that a referral to nephrology is to be initiated when patients reach a GFR of less than $30 \mathrm{ml} / \mathrm{min} / 1.73 \mathrm{~m}^{2}$ with or without diabetes, when they present with poorly-controlled hypertension even after treatment with at least 4 anti-hypertensive drugs, and when they reach an ACR of $30 \mathrm{mg} / \mathrm{mmol}$ (ACR category 3 ) or higher if presented along with haematuria [18].

A typical renal department provides inpatient and outpatient services covering general nephrology, pre- and post-dialysis review, low clearance clinics and transplant follow-up. The department is made up of a team of professionals from various disciplines including nephrologists (consultants and registrars), Clinical Nurse Specialists (CNS), renal councilors, dialysis nurses and dieticians. Joint clinics are typically run in parallel with endocrinologists for diabetic patients with CKD, with cardiologists to manage their heart failure and lastly with rheumatologists for patients with autoimmune multi systemic diseases.

A team of MDT managing CKD also includes vascular surgeons working together with interventional radiologists to get and maintain an intravenous access for dialysis. Interventional radiologists are valuable for the team since they can also perform some key procedures such as vascular access insertion or angioplasty. Some CT guided renal biopsy can be done by interventionist in tricky patients allowing the clinicians to treat the underlying causes. Vascular access CNS is very crucial in the maintenance of $\mathrm{AV}$ fistulae by reviewing dialysis patients' access on regular basis through quarterly transonics (device used to monitor access flow).

Furthermore, specialists from other disciplines are also integral to the renal MDT allowing various other disease aetiologies to be managed adequately. Urologists will help in patients with obstructive uropathy and histopathologists will review kidney biopsies for diagnosis of unclear causes of acute kidney injury or CKD.

\section{Role of MDT in Management of CKD}

\section{Anti-hypertensive therapy}

There is a $57 \%$ increased mortality from cardiovascular causes when patients reach a GFR of less than $60 \mathrm{~mL} / \mathrm{min}$ per $1.73 \mathrm{~m}^{2}$ [19]. Moreover, CKD patients have 5-10-fold increased risks of dying from other complications than progression into ESKD [20]. Keith DS, et al. [21] also published results from a longitudinal study which concluded that CKD patients are twice likely to die from CVD complications than develop ESKD. Thus, one of the roles of the consultant nephrologists is to prescribe anti-hypertensives accordingly, to monitor patients' blood pressure and possible other side effects arising from these therapies. Antagonists of renin-angiotensin-aldosterone system such as 
Angiotensin Converting-Enzyme Inhibitors (ACEIs) or Angiotensin II Receptor Blockers (ARBs) are the first-line choice of agents in CKD patients to reduce proteinuria which is the main culprit for disease progression [22]. ARBs and ACEIs can also lead to hyperkalaemia in CKD patients and therefore should be continuously monitored for their blood potassium levels.

\section{Management of anaemia}

One of the main functions of kidney is to produce Erythropoietin (EPO) which is a hormone stimulating red blood cell production. CKD patients typically present with normocytic normochromic anaemia due to impaired EPO production by the kidneys. Chronic anaemia in CKD is associated with reduced quality of life and increased mortality. There is a $29 \%$ increased chance of hospitalisation in patients with Haemoglobin $(\mathrm{Hb})$ of $<10 \mathrm{~g} / \mathrm{dl}$ than those with $\mathrm{Hb}$ between 11-12 $\mathrm{g} / \mathrm{dl}$ [23]. The iron, recombinant erythropoietin and its derivatives such as epoetin alfa, beta and darbepoetin alfa are commonly used in treatment of anaemia to decrease need to transfuse blood [24]. Chronic anaemia is normally managed by anaemia CNS who monitor patients' haematinics; iron levels, folic acid and Vitamin B12; and treat accordingly. In case of iron deficiency, they will arrange administering intravenous iron and Erythrocyte-Stimulating Agents (ESAs) to the patients, as prescribed by the nephrologists. They are also important in providing counselling sessions for pre-dialysis and dialysis patients informing them of local and national treatment guidelines to help decide their future treatment plans.

\section{Management of CKD mineral bone disease and gout}

The kidneys regulate how calcium and phosphate are absorbed in the intestine by regulating Vitamin-D metabolism in which they convert Vitamin-D to calcitriol (activated Vitamin D). CKD patients can present with abnormal serum calcium and phosphate concentrations along with decreased levels of active Vitamin D. As a result, osteoclast activity is enhanced by increasing resorbing and breaking down bone tissue, thereby causing CKD patients to suffer from increased bone pain, or bone fragility. Management of CKD mineral bone diseases include renal diets restricting phosphates, prescription of calcium or non-calcium phosphate-binders, and activated Vitamin $\mathrm{D}$ in those with low serum calcitriol levels $(>30 \mathrm{ng} / \mathrm{mL}$ ) and with normal parathyroid hormone levels [3]. In addition, CKD patients often have high uric acid level (hyperuricemia) which can lead to acute or chronic gout attacks. Uric acid lowering agents such as allopurinol and febuxostat are used to manage the hyperuricemia. Therefore, it is the role of the renal dieticians to offer guidance on which food to avoid and how to prepare an adequate meal for good renal health which often constitute foodstuffs low in phosphorous, calcium and potassium, along with offering support and help to lose weight, exercise more and quit smoking.

\section{Renal Replacement Therapy (RRT)}

There is no universal definition or endpoint of CKD at which ESKD can be diagnosed and RRT is to be established, since patients can have differing levels of co-morbidities. When the patients' eGFRs start to approach $15 \%$, they are consulted to discuss and make timely arrangement for further treatment plans including starting dialysis (either peritoneal or haemodialysis), kidney transplant or undertaking conservative treatment. However, a study supported by USA, National Kidney Foundation concluded that an acceptable surrogate endpoint should be when a decrease of eGFR of $30-40 \%$ within 2-3 years excluding causes of Acute Kidney Injury (AKI) [25]. Generally, GFR of less than $15 \mathrm{~mL} / \mathrm{min} / 1.73 \mathrm{~m}^{2}$ along with presentation of uraemic symptoms can warrant a consultation of RRT which involves either dialysis or kidney transplantation.

RRT takes up a huge portion of NHS kidney budget and there was an estimated total annual cost for both haemodialysis and peritoneal dialysis of $£ 505$ million and a total annual cost for all transplants was reported to be $£ 225$ million in 2009-2010 [1]. Having a strong MDT team behind the patients makes an important difference to ESKD patients regarding making life-changing decisions when considering RRT. There is a huge burden for patients to consider whether to enlist for RRT or undergo conservative management. A randomised control study carried out by Cooper BA, et al. [26] in Australia and New Zealand in 2010 (IDEAL study) did not find any difference or improvement in survival outcomes and mortality rates when stage V CKD patients were started on early or late dialytic treatments. Therefore, it is paramount that a patient needs to be educated and consulted on optimal timing of initiating dialysis, which modality of dialysis to choose, extent of time waiting for the matched donor kidney for transplantation and how to cope with immunosuppressants and post-transplant complications.

\section{Overall impact of MDT in CKD}

Incorporation of MDT in CKD care has been supported widely due to its positive outcomes from several studies published over the years. A study by Lin E, et al. [27] published in PLoS Med, found that MDT increased Quality Of Life Adjusted Years (QALYs) by 0.23 per person, when compared with non-MDT care. Cost-effectiveness of MDT has also been reported where MDT care leads to a reduction of $\$ 1931$ annually per patient from reduced RRT and emergent need for dialysis since MDT was shown to slow eGFR decline and infection-specific hospitalisation [28].

Furthermore, a prospective study led by Chen, et al. [29] in Taiwan, also reported that patients under MDT care had a $51 \%$ reduced mortality than patients under usual non-MDT care. Moreover, a holistic approach provided via MDT care ensures that one will be guaranteed with an individualised and targeted management plan including health education and awareness of nephrotoxic Nonsteroidal Anti-Inflammatory Drugs (NSAIDs) which are contraindicated in $\mathrm{CKD}$ patients since they can lead to severe renal injury and progression of CKD [30].

\section{Conclusion}

To conclude, CKD is a chronic disease associated with multiple comorbidities. To manage such a multi-faceted disease accordingly, only a MDT with professionals working towards the same goals and acting in the best interests of patients will be able to improve patients' quality of life and bring positive clinical outcomes. As CKD progresses, there is a need for a more collaborative effort between members of the MDT to address the increasing needs of the patients as they present with more complications. However, integration of such collaborative works may also need enhanced communication between colleagues to provide optimal care for the patients. Although effectiveness of providing a more integrated care via MDT has been established, determination of one optimal professional in an MDT has not been elucidated yet, requiring more randomised studies to follow.

\section{References}

1. NHS England (2017) Chronic kidney disease in England: the human and financial cost. London.

2. Public Health England (2014) Chronic kidney disease prevalence model. London. 
3. Kidney Disease: Improving Global Outcomes (KDIGO) CKD-MBD Update Work Group (2017) KDIGO 2017 Clinical Practice Guideline Update for the Diagnosis, Evaluation, Prevention, and Treatment of Chronic Kidney Disease-Mineral and Bone Disorder (CKD-MBD). Kidney Int Supp 7: 1-59.

4. Rahn KH, Heidenreich S, Bruckner D (1999) How to assess glomerular function and damage in humans. J Hypertens 17: 309-317.

5. Sandilands EA, Dhaun N, Dear JW, Webb DJ (2013) Measurement of renal function in patients with chronic kidney disease. $\mathrm{Br} J \mathrm{Clin}$ Pharmacol 76: 504-515.

6. Stevens LA, Coresh J, Greene T, Levey AS (2006) Assessing kidney function-measured and estimated glomerular filtration rate. $\mathrm{N}$ Engl J Med 354: 2473-2483.

7. Smith HW, Goldring W, Chasis H (1938) The measurement of the tubular excretory mass, effective blood flow and filtration rate in the normal human kidney. J Clin Invest 17: 263-278.

8. Florkowski CM, Chew-Harris JS (2011) Methods of estimating GFRdifferent equations including CKD-EPI. Clin Biochem Rev 32: 75-79.

9. National institute of diabetes and digestive and kidney disease (NIDDK) Estimating Glomerular Filtration Rate. NIDDK, National Institute of Health, USA.

10. Levey AS, Coresh J (2012) Chronic kidney disease. Lancet 379: 165 180.

11. Wyatt RJ, Julian BA (2013) IgA nephropathy. N Engl J Med 368: 2402 2414.

12. Neugarten J, Golestaneh L (2013) Gender and the prevalence and progression of renal disease. Adv Chronic Kidney Dis 20: 390-395.

13. Morton RL, Schlackow I, Mihaylova B, Staplin ND, Gray A, et al. (2016) The impact of social disadvantage in moderate-to-severe chronic kidney disease: an equity-focused systemic review. Nephrol Dial Transplant 31: 46-56.

14. Chang A, Kramer H (2012) CKD progression: a risky business. Nephrol Dial Transplant 27: 2607-2609.

15. Vanholder R, Baurmeister U, Brunet P, Cohen G, Glorieux G, et al. (2008) A bench to bedside view of uremic toxins. J Am Soc Nephrol 19: 863-870.

16. Kumar V, Abbas AK, Aster JC (2015) Robbins and Cotran pathologic basis of disease. 9 $^{\text {th }}$ Edition Elsevier/Saunders, Philadelphia, USA.

17. Rosa-Diez G, Gonzalez-Bedat M, Pecoits-Filho R, Marinovich S, Fernandez S, et al. (2014) Renal replacement therapy in Latin American end-stage renal disease. Clin Kidney J 7: 431-436.
18. National institute of health and care excellence (NICE) (2014) Chronic kidney disease in adults: assessment and management. NICE, London

19. Di Angelantonio E, Danesh J, Eiriksdottir G, Gudnason V (2007) Renal function and risk of coronary heart disease in general populations: new prospective study and systematic review. PLoS Med 4: e270.

20. Collins AJ, Foley RN, Gilbertson DT, Chen SC (2015) United States Renal Data System public health surveillance of chronic kidney disease and end-stage renal disease. Kidney Int Suppl 5: 2-7.

21. Keith DS, Nichols GA, Gullion CM, Brown JB, Smith DH (2004) Longitudinal follow-up and outcomes among a population with chronic kidney disease in a large managed care organization. Arch Intern Med 164: 659-663.

22. Tomson C, Taylor D (2015) Management of chronic kidney disease. Medicine 43: 454-461.

23. Locatelli F, Pisoni RL, Combe C, Bommer J, Andreucci VE, et al. (2004) Anaemia in haemodialysis patients of five European countries: association with morbidity and mortality in the Dialysis Outcomes and Practice Patterns of Study (DOPPS). Nephrol Dial Transplant 19: 121-132.

24. Webster AC, Nagler EV, Morton RL, Masson P (2017) Chronic kidney disease Lancet 389: 1238-1252.

25. Levey AS, Inker LA, Matsushita K, Greene T, Willis K, et al. (2014) GFR decline as an end point for clinical trials in CKD: a scientific workshop sponsored by the National Kidney Foundation and the US Food and Drug Administration. Am J Kidney Dis 64: 821-835.

26. Cooper BA, Branley P, Bulfone L, Collins JF, Craig JC, et al. (2010) A randomised, controlled trial of early versus late initiation of dialysis. N Engl J Med 363: 609-619.

27. Lin E, Chertow GM, Yan B, Malcolm E, Goldhaber-Fiebert JD (2018) Cost-effectiveness of multidisciplinary care in mild to moderate chronic kidney disease in the United States: A modelling study. PLoS Med 15: e1002532.

28. Chen PM, Lai TS, Chen PY, Lai CF, Yang SY, et al. (2015) Multidisciplinary care program for advanced chronic kidney disease: reduces renal replacement and medical costs. Am J Med 128: 68-76.

29. Chen YR, Yang Y, Wang SC, Chiu PF, Chou WY, et al. (2013) Effectiveness of multidisciplinary care for chronic kidney disease in Taiwan: a 3-year prospective cohort study. Nephrol Dial Transplant 28: 671-682.

30. Pazhayattil GS, Shirali AC (2014) Drug-induced impairment of renal function. Int J Nephrol Renovasc Dis 7: 457-468. 